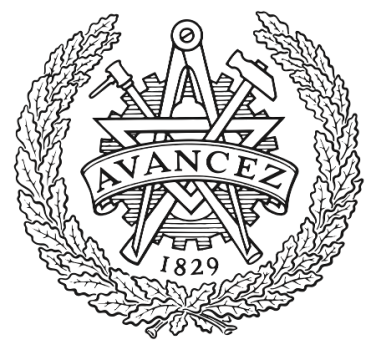

CHALMERS

UNIVERSITY OF TECHNOLOGY

\title{
A Wide Coverage S-Band Array with Dual Polarized Connected Bowtie Antenna Elements
}

Downloaded from: https://research.chalmers.se, 2023-04-26 02:46 UTC

Citation for the original published paper (version of record):

Khanal, P., Yang, J., Ivashina, M. et al (2019). A Wide Coverage S-Band Array with Dual Polarized Connected Bowtie Antenna Elements. 2019 IEEE International Symposium on Antennas and Propagation and USNC-URSI Radio Science Meeting: 2001-2002.

http://dx.doi.org/10.1109/APUSNCURSINRSM.2019.8888598

N.B. When citing this work, cite the original published paper. 


\title{
A Wide Coverage S-Band Array with Dual Polarized Connected Bowtie Antenna Elements
}

\author{
Prabhat Khanal ${ }^{1}$, Jian Yang ${ }^{1}$, Marianna Ivashina ${ }^{1}$, Anders Hook ${ }^{2}$ and Ruoshan Luo ${ }^{2}$ \\ ${ }^{1}$ Department of Electrical Engineering, Chalmers University of Technology, Gothenburg, Sweden \\ prabhat@chalmers.se, jian.yang@chalmers.se, marianna.ivashina@chalmers.se \\ 2 SAAB AB, Gothenburg, Sweden \\ anders.hook@saabgroup.com,ruoshan.luo@saabgroup.com
}

\begin{abstract}
A low profile dual polarized bowtie antenna element is studied for an S-band $(3 \mathrm{GHz})$ wide-scan application in a large-scale (nearly an infinitely large) uniformly excited phased array. The study shows that the array with such elements, when connected, is capable of scanning up to $\pm 75^{\circ}$ at E-plane and $\pm 15^{\circ}$ at $\mathrm{H}$-plane for horizontal and vertical polarizations respectively. The bandwidth of the infinite array with such elements across these scan angles is $10 \%$ and $25 \%$ for the active reflection coefficient of $-10 \mathrm{~dB}$ and $-5 \mathrm{~dB}$ respectively.
\end{abstract}

Keywords- A wide scan array, connected array, bowtie array, phased array, dual polarization.

\section{INTRODUCTION}

One of the main development trends in the aerospace industry is to improve the aerodynamic characteristics of an aircraft using lightweight structures to reduce fuel consumption and impact on the environment. Multifunctional structures with integrated systems like fuselage integrated antenna systems may provide, and improve sensor performance due to the great flexibility in the mounting position [1], [2]. The integrated solutions could be used for applications like air surveillance, satellite communication, electronics warfare, etc.

This paper presents a new antenna element which can potentially be used in such fuselage integrated antenna systems. The element is a dual polarized bowtie, and it is studied in an infinite connected array environment. In a connected array, the adjacent antenna elements are physically or electrically connected to each other, and hence are strongly mutually coupled in order to operate over a wide frequency band [3], [4]. In arrays, this connected element approach is considered as a suitable solution for wide-band, wide-scan applications [5]. This concept has been used for the dipole [5], Vivaldi [6] and slot [7] antenna elements. In this paper, the concept of connected array is implemented with a dual polarized bowtie element to achieve a relatively low profile $\left(\sim 0.35 \lambda_{\text {max }}\right)$, wide scan $\left( \pm 75^{\circ}\right.$ at E-plane and $\pm 15^{\circ}$ at H-plane) antenna array.

\section{ANTENNA CHALLENGES}

The studied antenna structure is required to endure very tough environmental conditions. For example, wind pressure on the fuselage can reach up to around $4,000 \mathrm{~Pa}$, static loads to $2 \mathrm{~g}$, and bending moment to $30,000 \mathrm{Nm}$. The operating temperature has a wide range from $-65^{\circ} \mathrm{C}$ to $+55^{\circ} \mathrm{C}$.
The array is targeted to have a high gain and wide beam scan angles with $20 \times 200$ (vertical $\times$ horizontal) antenna elements operating at $\mathrm{S}$-band $(3 \mathrm{GHz})$, while enabling the integration with a low profile conformal radome to the fuselage. The required active reflection coefficients $\left(\Gamma_{a c t}\right)$ and the relative bandwidth at different operating conditions are given in Table I.

TABLE I: Electrical specifications of the antenna array

\begin{tabular}{|c|c|c|c|}
\hline Operating conditions & Bandwidth & $\Gamma_{a c t}$ & Scan angles \\
\hline Performance & $10 \%$ & $-10 \mathrm{~dB}$ & $\begin{array}{l} \pm 75^{\circ} \text { at E-plane } \\
\pm 15^{\circ} \text { at H-plane }\end{array}$ \\
\hline Function & $25 \%$ & $-5 \mathrm{~dB}$ & $\begin{array}{l} \pm 75^{\circ} \text { at E-plane } \\
\pm 15^{\circ} \text { at H-plane }\end{array}$ \\
\hline
\end{tabular}

\section{ANTENNA DESIGN}

Fig. 1 shows the antenna element, antenna ports, crosssection view of the element and the array with the elements in the connected infinite boundary condition. The dual polarized element is realized by two orthogonal bowties which are above the ground plane supported by two pairs of pillars, each pair for each bowtie. One pillar in each pair is a coaxial feed line whose outer conductor is connected to the ground plane at the one end and to one arm of a bowtie at the other end, and whose inner connector is connected to the other arm of the same bowtie via a metal microstrip line on a small piece of $\mathrm{PCB}$. The bowtie arm connected with the inner conductor is also connected to the ground via the second pillar (solid) in the same pair of the pillars. As in Fig. 1c, one of these two strips (for each bowtie) is in the top layer and the other one is in the middle layer of the PCB. These pillars functions as baluns that transform the single-ended antenna ports to the differential ports of the bowties.

This antenna element has a wide bandwidth and a broad embedded radiation beam pattern, which are essential for large scanning antenna array. The element is defined by several geometric and electric parameters, which introduces a few parametric freedoms for optimizing the scan reflection coefficients, especially at wide scan angles. The connected array structure is mainly used to improve wide scan capabilities. Furthermore, it also makes the array mechanically strong and resilient to strong vibrations because the elements support each other, and hence make a more stable structure. 


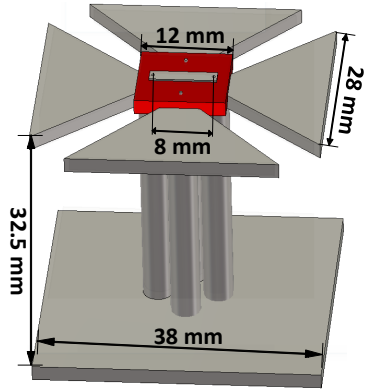

(a)

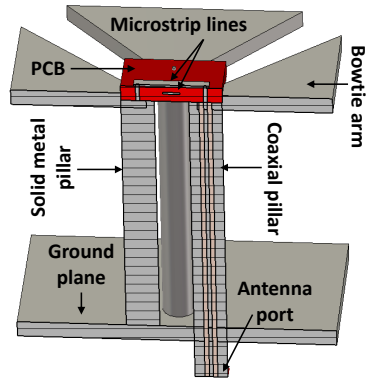

(c)

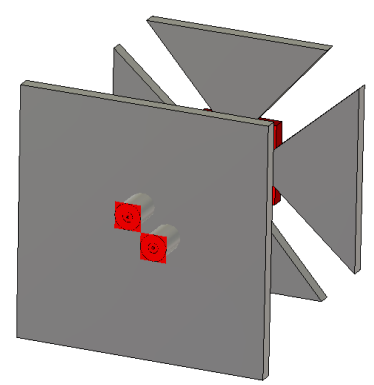

(b)

(d)

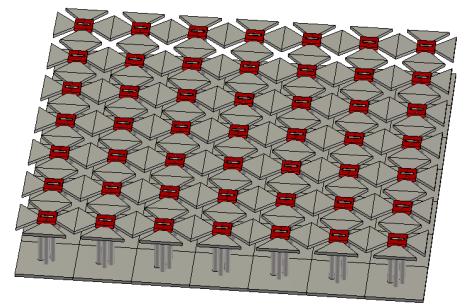

Fig. 1: The cross bowtie antenna element; (a) structure, (b) antenna ports, (c) cross-section and (d) array configuration

\section{RESUlTS}

The element was optimized for the uniformly excited infinite phased array as in [6]. The optimization strategy was to first optimize the element at a wide scan angle $\left(60^{\circ}\right.$ in this case) in order to obtain a good performance over the scanning angles from $0^{\circ}$ to $\pm 75^{\circ}$. The active reflection coefficients of the uniformly excited infinite phased array at various scan angles for horizontal polarization and vertical polarization are shown in Fig. 2a and $2 \mathrm{~b}$ respectively.

Here, it can be seen that the element fulfills the requirements in Table I. The active reflection coefficient is $-10 \mathrm{~dB}$ for $10 \%$ bandwidth and $-5 \mathrm{~dB}$ for $25 \%$ bandwidth at scan angles up to $\pm 75^{\circ}$ at E-plane and $\pm 15^{\circ}$ at H-plane. However, the performance at the center frequency (the nadirs) for horizontal polarization and vertical polarization are different by about $200 \mathrm{MHz}$ apart from each other. This is due to the slight asymmetry in the feeding structure (see the PCB in Fig. 1c) of the orthogonal bowties. The optimization procedure was only carried out at the horizontal polarization. It could be improved by additional optimization iterations.

The element was also studied in a large finite $(7 \times 7$ elements) uniformly excited phased array. As shown in Fig. 3 the active reflection coefficient of the central element of the array is also $-10 \mathrm{~dB}$ for $10 \%$ bandwidth and $-5 \mathrm{~dB}$ for $25 \%$ bandwidth at scan angles up to $\pm 55^{\circ}$ at E-plane and $\pm 15^{\circ}$ at H-plane. It is important to notice that the $7 \times 7$ elements array has a narrower scan angle range compared to the infinite array as expected. This is because each element is strongly coupled to its adjacent elements and the array is relatively small, so the active reflection coefficient of the elements in the middle are affected by the edge truncation effects. Similar to the infinite array (see Fig. 2), the center frequency of horizontal polarization and vertical polarization of the central element of the $7 \times 7$ elements array are also different and about $200 \mathrm{MHz}$ apart from each other.

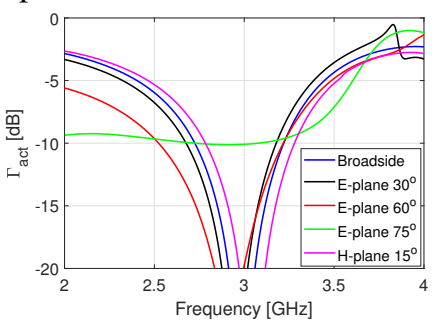

(a)

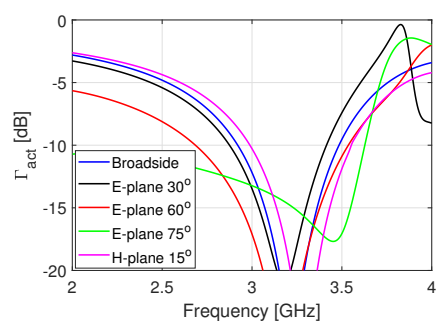

(b)
Fig. 2: The active reflection coefficients of the uniformly excited infinite phased array of connected cross bowtie antenna elements for a) horizontal and b) vertical polarizations.

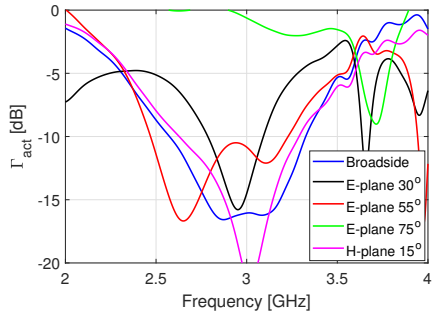

(a)

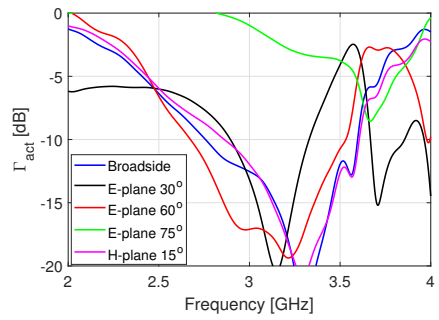

(b)
Fig. 3: The active reflection coefficient of the uniformly excited $7 \times 7$ elements phased array of connected cross bowtie antenna elements for a) horizontal and b) vertical polarizations.

\section{CONCLUSION}

The cross bowtie antenna element has promising simulation results in terms of a wide scan range for infinitely large arrays. In the same time, it exhibits possibility for the fuselage integrated antenna array design.

\section{REFERENCES}

[1] J. Yee and W. Furlong, "An extremely lightweight fuselage-integrated phased array for airborne applications," IEEE Transactions on Antennas and Propagation, vol. 29, pp. 178-182, January 1981.

[2] M. Urcia and D. Banks, "Structurally integrated phased arrays," in 2011 Aerospace Conference, pp. 1-8, March 2011.

[3] R. C. Hansen, "Current induced on a wire: implications for connected arrays," IEEE Antennas and Wireless Propagation Letters, vol. 2, pp. 288 289, 2003.

[4] B. A. Munk, Finite Antenna Arrays and FSS. A John Wiley \& Sons, Inc Publication, 2005.

[5] D. Cavallo, G. Gerini, R. Bolt, D. Deurloo, R. Grooters, A. Neto, G. Toso, and R. Midthassel, "Ku-band dual-polarized array of connected dipoles for satcom terminals: Theory and hardware validation," in 2013 7th European Conference on Antennas and Propagation (EuCAP), pp. 459460, April 2013.

[6] R. Maaskant, M. V. Ivashina, O. Iupikov, E. A. Redkina, S. Kasturi, and D. H. Schaubert, "Analysis of large microstrip-fed tapered slot antenna arrays by combining electrodynamic and quasi-static field models," IEEE Transactions on Antennas and Propagation, vol. 59, pp. 1798-1807, June 2011.

[7] D. Cavallo, W. H. Syed, and A. Neto, "Connected-slot array with artificial dielectrics: A 6 to 15 ghz dual-pol wide-scan prototype," IEEE Transactions on Antennas and Propagation, vol. 66, pp. 3201-3206, June 2018. 